\title{
Measurement of the Lower Lid Margin Thickness by Anterior Segment Optical Coherence Tomography
}

\author{
Da-Hu Wang ${ }^{\text {a }}$ Zhao-Yan Li ${ }^{b}$ Xin-Quan Liu ${ }^{a}$ \\ ${ }^{a}$ Department of Ophthalmology, LongHua Hospital Shanghai University of Traditional Chinese Medicine, \\ Shanghai, China; ${ }^{\mathrm{b}}$ Longhua Clinical Medical College, Shanghai University of Traditional Chinese Medicine, \\ Shanghai, China
}

\section{Keywords}

Meibomian gland dysfunction · Lower lid margin thickness · Anterior segment optical coherence tomography

\begin{abstract}
Purpose: To verify the feasibility of using anterior segment optical coherence tomography (AS-OCT) to measure the lower lid margin thickness (LLMT) from posterior lash line to the mucocutaneous junction. Methods: This was a prospective, single-center, observational study. Fifty-six volunteers between 20 and 79 years old with normal healthy eyes were included in this study. Using AS-OCT to measure the LLMT at the middle position was separately performed by two operators under the same conditions. Results: The mean age of 56 subjects ( 24 males and 32 females) was $45.4 \pm 15.7$ years. The LLMT measured by AS-OCT between two operators was $0.86 \pm 0.23 \mathrm{~mm}$ and $0.85 \pm 0.22 \mathrm{~mm}$, respectively. The reliability and repeatability between two operators were evaluated by intraclass correlation coefficient (ICC $=0.97)$. Based on one operator's measurements, the LLMT was $0.70 \pm 0.10$ $\mathrm{mm}$ at ages 20-29, $0.68 \pm 0.09 \mathrm{~mm}$ at ages 30-39, $0.94 \pm 0.12$ $\mathrm{mm}$ at ages $40-49,0.93 \pm 0.20 \mathrm{~mm}$ at ages 50-59, $1.03 \pm 0.15$
\end{abstract}

karger@karger.com www.karger.com/ore

(C) 2020 S. Karger AG, Basel

Karger! $\mathrm{mm}$ at ages $60-69$, and $1.24 \pm 0.19 \mathrm{~mm}$ at ages $70-79$, which indicated a significant positive correlation with age $\left(r_{s}=0.74\right.$, $p<0.001)$. In addition, there was a significant difference in the LLMT between genders $(0.94 \pm 0.26$ vs. $0.80 \pm 0.19 \mathrm{~mm}$, $p=0.03)$. Conclusions: A good agreement between two operators indicated that it seemed to be feasible to use AS-OCT to measure the LLMT. Additionally, we found that the LLMT appeared to be closely related to age and gender.

(c) 2020 S. Karger AG, Basel

\section{Introduction}

The free edge of the eyelid is called the lid margin, which is divided into anterior lid margin and posterior lid margin according to the gray line $[1,2]$. Thickening of the lid margin is a common feature of advanced meibomian gland dysfunction (MGD) [3-5], which is closely associated with evaporative dry eye $[2,6-8]$, but it is difficult to measure because of rounding of the posterior lid margin.

Da-Hu Wang and Zhao-Yan Li contributed equally to this work. 


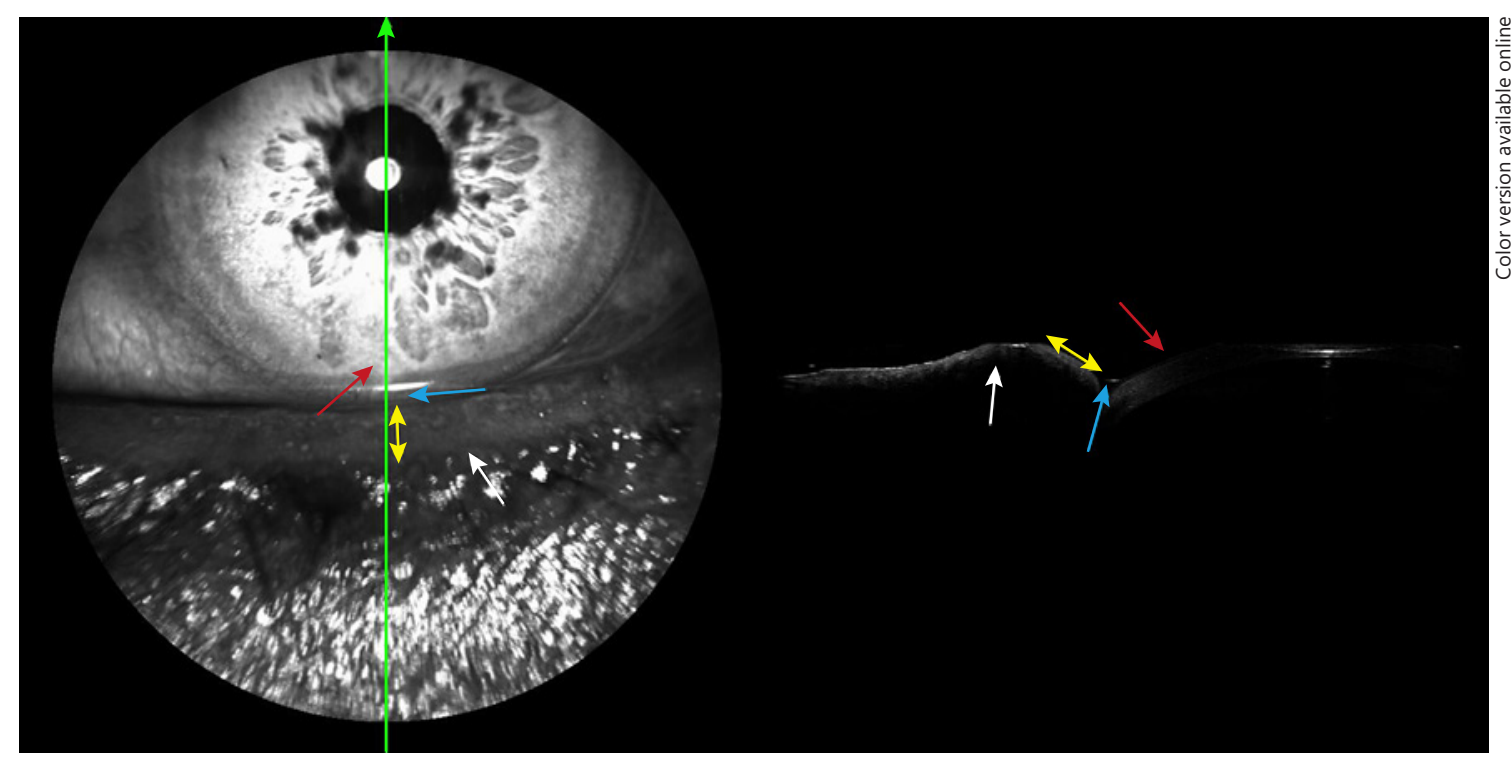

Fig. 1. A representative AS-OCT image of measuring the lower lid margin thickness. The long green arrow stands for the optimal position of OCT scanning, the two white arrows stand for the lower lid, the two blue arrows stand for the lower tear meniscus, the two red arrows stand for the cornea, and the two yellow double arrows stand for the lower lid margin thickness (posterior lash line to anterior edge of the tear meniscus or mucocutaneous junction).

However, in later-stage MGD, there were other downstream effects of the disease (e.g., telangiectasia, abnormal orifices, irregular lid margin), which offered easier assessment for diagnosis than measurement of the lid margin thickness. But in early-stage MGD or asymptomatic MGD (preclinical stage), its clinical features may not be obvious to the observers. At this stage, it may be diagnosed by the meibomian gland expression or meibography [3]. As to whether there are early changes in the lid margin thickness, there is no relevant published literature at present.

In addition, changes in the lid margin thickness may also be seen in some diseased eyes, such as blepharitis, lidwiper epitheliopathy, and conjunctivochalasis. Therefore, the measurement of the lid margin thickness could be helpful to evaluate several different eye disorders. Up to date, several measurements such as ruler, vernier micrometer, and Scheimpflug camera [9-11] have been used to measure the lid margin thickness from the posterior lash line to the posterior margin, which is a relatively constant feature of the lid margin [5], but these measurements have some limitations in clinical practice due to the inconvenience and/or complexity of the instruments.

Optical coherence tomography (OCT) has been clinically used for nearly 30 years since it was introduced for medical purpose in 1991 [12]. At present, anterior segment optical coherence tomography (AS-OCT) plays an important role in ophthalmology, which includes cornea, the anterior chamber and angle, the aqueous outflow system, ocular surface, and dry eye disease [13]. Compared with the traditional methods such as ruler and vernier micrometer $[9,10]$, the main advantages of AS-OCT include the rapid, simple, convenient, and noninvasive in vivo imaging of the eye structures, while providing accurate quantitative measurements and imaging data. Therefore, in this study, we try to introduce AS-OCT to measure the lid margin thickness at the middle position of the lower lid (Fig. 1) and evaluate its reliability.

\section{Materials and Methods}

\section{Subjects}

This was a prospective, single-center, observational study. Fiftysix volunteers ( 24 males and 32 females) between 20 and 79 years with normal healthy eyes were included in this study. The study was conducted in strict accordance with the principles of the Helsinki Declaration under the guidance of Ethics committee of the Longhua Hospital Shanghai University of Traditional Chinese Medicine (No. 2019LCSY031). Each subject signed informed consent for the study.

Study exclusion criteria were Ocular Surface Disease Index scores higher than 12 points [3]; lid structural abnormalities; irregular lid margins; acne rosacea; eyelid entropion and trichiasis; eyelid tumor; no eyelashes or central eyelashes loss in lower lid; conjunctivo chalasis; nystagmus; paralytic strabismus; ocular allergies; topical antiglaucoma therapy; ocular infection and/or in- 
Table 1. Demographic characteristics for subjects

\begin{tabular}{|c|c|c|c|c|c|c|c|}
\hline & \multicolumn{7}{|c|}{ Age group } \\
\hline & $20-29$ & $30-39$ & $40-49$ & $50-59$ & $60-69$ & $70-79$ & Total \\
\hline Subjects & 10 & 16 & 8 & 8 & 9 & 5 & 56 \\
\hline \multicolumn{8}{|l|}{ Age, years } \\
\hline Mean \pm SD & $26.5 \pm 1.6$ & $33.9 \pm 3.3$ & $45.2 \pm 2.4$ & $54.3 \pm 2.2$ & $63.6 \pm 3.4$ & $73.2 \pm 2.5$ & $45.4 \pm 15.7$ \\
\hline Median & 27 & 32.5 & 45.5 & 54.0 & 63.0 & 73.0 & 43.0 \\
\hline Min., max. & 24,28 & 30,39 & 42,48 & 52,58 & 60,69 & 70,77 & 24,77 \\
\hline \multicolumn{8}{|l|}{ Gender, $n(\%)$} \\
\hline Male & $3(30.0)$ & $6(37.5)$ & $4(50)$ & $2(25.0)$ & $5(55.5)$ & $4(80.0)$ & $24(42.9)$ \\
\hline Female & $7(70.0)$ & $10(62.5)$ & $4(50)$ & $6(75.0)$ & $4(44.5)$ & $1(20.0)$ & $32(57.1)$ \\
\hline
\end{tabular}

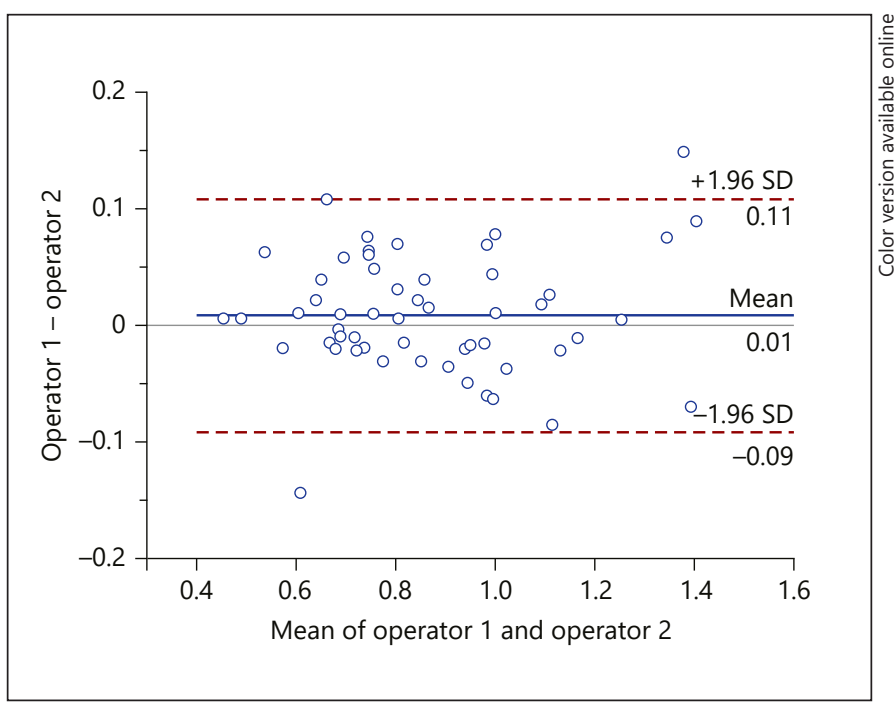

Fig. 2. Agreement analysis between two operators. Bland-Altman plots demonstrated that 53 of 56 spots were within 95\% limits of agreement (95\% LoA). The maximum difference was $0.11 \mathrm{~mm}$ within $95 \%$ LoA and the arithmetic mean was $0.01 \mathrm{~mm}$.

flammation; had worn contact lens; had penetrating intraocular surgery or ocular surgery; used intense pulsed light or isotretinoin (accutane); was pregnant or lactating.

\section{Measurements of Lid Margin Thickness}

The thicknesses of the lower lid margin were measured by ASOCT (Spectralis OCT, Heidelberg Engineering, Germany) for each subject, and the protocol process was basically the same as the measurement of tear meniscus height [14]. The specific steps were as follows. During the process, subjects were seated in front of AS-OCT, their heads were comfortably placed on the chin and forehead rested, and they were asked to look straight ahead, then AS-OCT was used to measure the lower lid margin thickness (LLMT) at the middle position (see online suppl. video at www. karger.com/doi/10.1159/000507221, which demonstrates the measurement of LLMT with AS-OCT). Measurements were respectively performed by two operators under the same conditions.
Because the optical properties of AS-OCT technique determines its poor tissue penetration, we cannot measure the full thickness of lid margin. Then, in order to get an accurate measurement, we only measured the LLMT from posterior lash line to anterior edge or outer edge of the tear meniscus, i.e., the keratinized skin width. We all knew that the palpebral border of lid margin in healthy people corresponded with the mucocutaneous junction (MCJ), i.e., the position of anterior edge of tear meniscus. When measuring the LLMT with AS-OCT, the boundary of MCJ was not very clear in the image, so we regarded the anterior edge of tear meniscus to replace the MCJ as the posterior boundary of the lid margin skin. In this study, without specific explanation, the LLMT we measured referred to the keratinized lid margin surface width, rather than the full thickness of lid margin.

\section{Statistical Analysis}

Only the right eye data were analyzed, as there was no statistically significant difference between the results of the right and left eyes. All data were presented as the mean \pm standard deviations. Statistical analyses were performed with Student's $t$ tests, intraclass correlation coefficient (ICC), Spearman's rank correlation coefficient, and Bland-Altman plots. $p<0.05$ was considered significant. Data analyses were performed with SPSS 18.0 software (SPSS Inc., Chicago, IL, USA).

\section{Results}

Fifty-six subjects ( 24 males and 32 females) were enrolled in the study. The mean age was $45.4 \pm 15.7$ years, with a range from 24 to 77 years. The detailed results of the demographic characteristics are presented in Table 1. The LLMT measured by AS-OCT between two operators was $0.86 \pm 0.23 \mathrm{~mm}$ and $0.85 \pm 0.22 \mathrm{~mm}$, respectively. The test of inter-operator variation showed that there was a good reliability and repeatability between two operators (ICC $=0.97, p=0.22$ ) and Bland-Altman plots also demonstrated a good agreement between two operators (Fig. 2). Based on one operator's measurements, the 


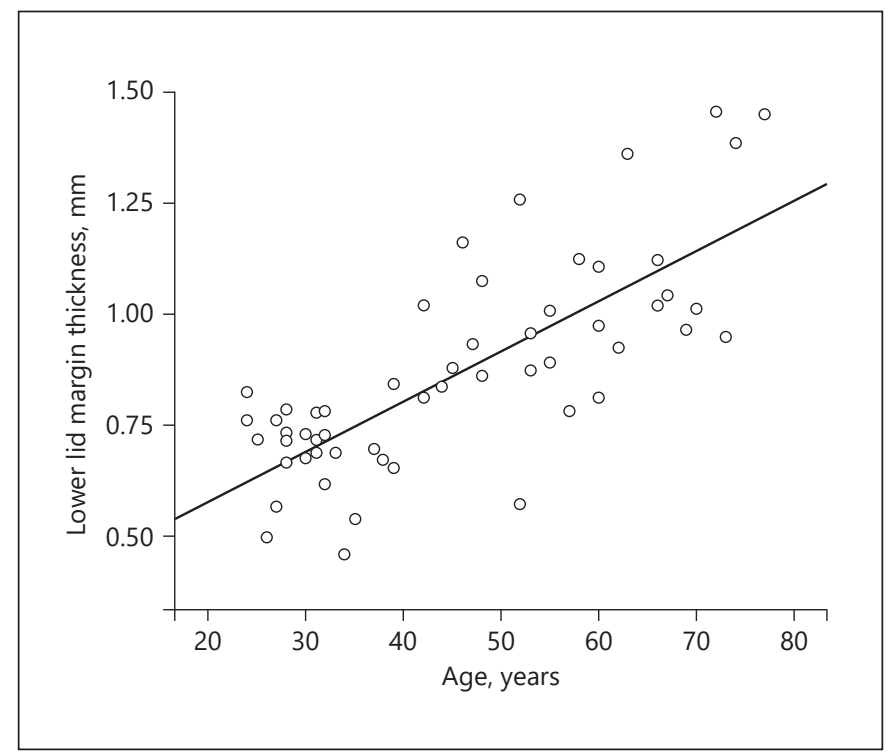

Fig. 3. Correlation between the lower lid margin thickness and age. The lower lid margin thickness was significantly positively correlated with age $\left(r_{\mathrm{s}}=0.74, p<0.001\right)$.

LLMT at the middle position was $0.70 \pm 0.10 \mathrm{~mm}$ at ages $20-29,0.68 \pm 0.09 \mathrm{~mm}$ at ages $30-39,0.94 \pm 0.12 \mathrm{~mm}$ at ages $40-49,0.93 \pm 0.20 \mathrm{~mm}$ at ages $50-59,1.03 \pm 0.15 \mathrm{~mm}$ at ages $60-69$, and $1.24 \pm 0.19 \mathrm{~mm}$ at ages $70-79$. In addition, the scatterplots and Spearman's rank correlation coefficient demonstrated that the LLMT was positively correlated with age $\left(r_{\mathrm{s}}=0.74, p<0.001\right.$; Fig. 3$)$. The average age between sexes was not significantly different ( 49.3 \pm 16.5 vs. $42.5 \pm 14.6$ years, $p=0.11)$, but there was a significant difference in the LLMT between genders $(0.94 \pm$ 0.26 vs. $0.80 \pm 0.19 \mathrm{~mm}, p=0.03)$. No adverse events were detected in all subjects after the measurements.

\section{Discussion}

MGD is a common disease in clinical practice. Population-based studies suggested that the incidence of MGD was higher in Asia, with a reported prevalence of $46.2 \%$ to $69.3 \%[6,15-17]$. In a study conducted in the United States and Europe, more than $80 \%$ of dry eye patients were found to have MGD [18]. The existing literature showed that the prevalence of MGD increased with age $[15,19,20]$, and MGD also occurred in young people [21-23]. As a summary of the above literature, high prevalence and younger trend of MGD suggest that it will be important to identify which preclinical features of early-stage MGD or asymptomatic MGD might be a predictor of progressive disease, and early intervention may thwart MGD. In addition to the meibomian gland expression or meibography [3], we also attempted to measure the LLMT with AS-OCT to detect the presence of MGD, so as to provide a certain clinical guidance for identifying and treating MGD earlier in the disease process.

Although it was difficult to measure the lid margin thickness, people were still working on it in several different ways. Hykin and Bron [9] measured the lid margin thickness at the free edge including the wet part of the eyelid (mucosa) with a vernier micrometer. The lid margin thickness in adults was $1.95 \pm 0.07 \mathrm{~mm}$ in the upper lid and $1.87 \pm 0.06 \mathrm{~mm}$ in the lower lid, with no significant difference between sexes. Meanwhile, Bron et al. [5] reported that the normal lid margin from its posterior border to the posterior lash line was approximately $2.5 \mathrm{~mm}$ wide in the adults. He [10] suggested that the lid margin thickness in Chinese (15-80 years) measured by a small ruler was approximately $2.0 \mathrm{~mm}$ in the upper lid and 1.5 $\mathrm{mm}$ in the lower lid. In addition, Pult et al. [11] used a Scheimpflug camera to measure the lid margin thickness in 15 adults, with no significant difference between the upper lid $(1.8 \mathrm{~mm})$ and the lower lid $(1.7 \mathrm{~mm})$. In this study, the LLMT measured by AS-OCT was less than that reported in the foregoing literature [5, 9-11]. One major reason for this discrepancy was that the LLMT we measured only included the width of the keratinized lid margin surface, excluding the marginal mucous membrane and MCJ. It should be emphasized that for the MCJ, its width varied due to different measurements, ranging from 0.09 to $0.3 \mathrm{~mm}$ [24-28].

According to reports in the literature $[2,9]$, the lid margin thickened with age over the first 20 years of life due to enlargement of the orbicularis and meibomian glands, but Hykin and Bron [9] suggested that the lid margin thickness in adults did not change with age. This conclusion may not be very well understood, because agerelated changes in the lid margin anatomy had been described in the previous studies $[5,9,19]$. Contrary to the conclusions drawn by Hykin and Bron [9], our present study showed that the LLMT in adults also seemed to be linked with age, which further supported the theory that the prevalence of MGD, including asymptomatic MGD, increased with age $[15,19,20,29-31]$. Specifically, in this study, the LLMT in young adults aged 20 to 39 was relatively stable, then increased with age (over 40 years).

The average thickness of the lower lid margin in men was greater than that in women $(p=0.03)$, which was inconsistent with the observations of Hykin and Bron [9]. 
At present, the relation between the LLMT and sex is not clear, but there were some notable studies regarding the association between changes in the meibomian glands and sex $[15,19,30-41]$. Siak et al. [32] showed that a higher proportion of men had MGD compared with women across all age groups $(p<0.001)$. Den et al. [19] also suggested that a high prevalence of abnormal lid margin and meibomian gland dropout was observed more frequently in men compared with women ( $\geq 70$ years). However, Lin et al. [15] revealed that women with dry eye symptoms showed a higher prevalence of anatomically abnormal meibomian glands than men $(p=0.078)$. Some laboratory studies [34-41] have found that abnormal lid margin and/or meibomian glands may be related to sex hormones. All the above-mentioned studies indicated a controversial association between MGD and sex, which still needs further study. Additionally, the lower lid margin in outdoor workers may be directly exposed to more ultraviolet radiation in sunlight [42-44], which may have a pathological impact on the lower eyelid disease, so labor distribution may have been hypothesized to explain sex differences [44].

In this pilot study, we innovatively used AS-OCT with the advantages of being easier, rapid, and noninvasive to measure the LLMT - the keratinized skin width. A good agreement between two operators showed the measurements of LLMT with AS-OCT were reproducible, which might provide a new insight for some eyelid disorders in the future. For instance, establishing a normal range of the LLMT for different age groups may be helpful for a quantitative analysis for early-stage MGD. In addition, we found that age and gender were significant factors affecting the LLMT in this study, despite the small sample size. Finally, it will be the focus of our future research to explore the relation between the LLMT and eyelid pressure, MGD.

However, there were some limitations in using ASOCT to measure the LLMT in our study including the following. (1) This study only measured the keratinized lid margin surface width from posterior lash line to the MCJ, not the full thickness of lid margin. (2) This study only measured the lid margin thickness in the lower lid, not the upper lid. (3) Once the images were not well centralized, then the measurement of LLMT would encounter difficulties. (4) We did not investigate associations between the lid margin thickness and clinical signs, systemic diseases. (5) A larger sample size and multicenter study would be helpful for better verifying the feasibility of ASOCT. Further studies are warranted to provide more information.
In conclusion, a good agreement between two operators indicated that noninvasive AS-OCT seemed to be a reliable method and might be useful for investigating the LLMT in normal and some eyelid-related diseases in the future, especially in early-stage MGD. In addition, we found that the LLMT appeared to be closely related to age and gender.

\section{Acknowledgements}

This work was supported by the National Natural Science Foundation of China (grant number: 81574028). We would like to acknowledge Xiao Wang and Cecilia Zhang for their English instructions.

\section{Statement of Ethics}

The study was conducted in strict accordance with the principles of the Helsinki Declaration under the guidance of Ethics committee of the Longhua Hospital Shanghai University of Traditional Chinese Medicine. Each subject signed informed consent for the study.

\section{Disclosure Statement}

The authors have no conflicts of interest to declare.

\section{Author Contributions}

D.-H. Wang conceived and designed the study, with input from X.-Q. Liu. D.-H. Wang and Z.-Y. Li analyzed the data, and wrote the initial manuscript draft. X.-Q. Liu actively reviewed and revised the manuscript, and all authors approved the final submitted manuscript.

\section{References}

1 Nelson JD, Shimazaki J, Benitez-del-Castillo JM, Craig JP, McCulley JP, Den S, et al. The international workshop on meibomian gland dysfunction: report of the definition and classification subcommittee. Invest Ophthalmol Vis Sci. 2011 Mar;52(4):1930-

2 Foulks GN, Bron AJ. Meibomian gland dysfunction: a clinical scheme for description, diagnosis, classification, and grading. Ocul Surf. 2003 Jul;1(3):107-26.

3 Tomlinson A, Bron AJ, Korb DR, Amano S, Paugh JR, Pearce EI, et al. The international workshop on meibomian gland dysfunction: report of the diagnosis subcommittee. Invest Ophthalmol Vis Sci. 2011 Mar;52(4):200649.
Wang/Li/Liu 
4 Asia Dry Eye Association China Branch, Ocular Surface and Tear Disease Group of Ophthalmology Specialized Committee of the Cross-Strait Medical Exchange Association. Chinese Expert Consensus on the Diagnosis and Treatment of Meibomian Gland Dysfunction (2017) [Chinese]. Zhonghua Yan Ke Za Zhi. 2017 Sep;53(9):657-61.

5 Bron AJ, Benjamin L, Snibson GR. Meibomian gland disease. Classification and grading of lid changes. Eye (Lond). 1991;5(Pt 4): 395-411.

6 Shimazaki J, Sakata M, Tsubota K. Ocular surface changes and discomfort in patients with meibomian gland dysfunction. Arch Ophthalmol. 1995 Oct;113(10):1266-70.

7 Goto E, Monden Y, Takano Y, Mori A, Shimmura S, Shimazaki J, et al. Treatment of noninflamed obstructive meibomian gland dysfunction by an infrared warm compression device. Br J Ophthalmol. 2002 Dec;86(12): 1403-7.

8 Nichols KK. The international workshop on meibomian gland dysfunction: introduction. Invest Ophthalmol Vis Sci. 2011 Mar;52(4): 1917-21.

9 Hykin PG, Bron AJ. Age-related morphological changes in lid margin and meibomian gland anatomy. Cornea. 1992 Jul;11(4):33442.

$10 \mathrm{He} \mathrm{L}$. [Measurement of the thickness of the normal lid margin in Chinese] [Chinese]. Zhonghua Yan Ke Za Zhi. 1982 May;18(3): 178.

11 Pult H, Korb DR, Murphy PJ, Riede-Pult BH, Blackie C. A new model of central lid margin apposition and tear film mixing in spontaneous blinking. Cont Lens Anterior Eye. 2015 Jun;38(3):173-80.

12 Huang D, Swanson EA, Lin CP, Schuman JS, Stinson WG, Chang W, et al. Optical coherence tomography. Science. 1991 Nov; 254(5035):1178-81.

13 Ang M, Baskaran M, Werkmeister RM, Chua J, Schmidl D, Aranha Dos Santos V, et al. Anterior segment optical coherence tomography. Prog Retin Eye Res. 2018 Sep;66:132-56.

14 Park DI, Shin HM, Lee SY, Lew H. Tear production and drainage after botulinum toxin $\mathrm{A}$ injection in patients with essential blepharospasm. Acta Ophthalmol. 2013 Mar; 91(2):e108-12.

15 Lin PY, Tsai SY, Cheng CY, Liu JH, Chou P, Hsu WM. Prevalence of dry eye among an elderly Chinese population in Taiwan: the Shihpai Eye Study. Ophthalmology. 2003 Jun; 110(6):1096-101.

16 Lekhanont K, Rojanaporn D, Chuck RS Vongthongsri A. Prevalence of dry eye in Bangkok, Thailand. Cornea. 2006 Dec;25(10): $1162-7$.

17 Jie Y, Xu L, Wu YY, Jonas JB. Prevalence of dry eye among adult Chinese in the Beijing Eye Study. Eye (Lond). 2009 Mar;23(3):68893.
18 Lemp MA, Crews LA, Bron AJ, Foulks GN, Sullivan BD. Distribution of aqueous-deficient and evaporative dry eye in a clinic-based patient cohort: a retrospective study. Cornea. 2012 May;31(5):472-8.

19 Den S, Shimizu K, Ikeda T, Tsubota K, Shimmura S, Shimazaki J. Association between meibomian gland changes and aging, sex, or tear function. Cornea. 2006 Jul;25(6):651-5.

20 Arita R, Itoh K, Inoue K, Amano S. Noncontact infrared meibography to document agerelated changes of the meibomian glands in a normal population. Ophthalmology. 2008 May;115(5):911-5.

21 Gupta PK, Stevens MN, Kashyap N, Priestley Y. Prevalence of Meibomian Gland Atrophy in a Pediatric Population. Cornea. 2018 Apr; 37(4):426-30

22 Wang X, Lu X, Yang J, Wei R, Yang L, Zhao $S$, et al. Evaluation of Dry Eye and Meibomian Gland Dysfunction in Teenagers with Myopia through Noninvasive Keratograph. J Ophthalmol. 2016;2016:6761206.

23 Mizoguchi T, Arita R, Fukuoka S, Morishige N. Morphology and Function of Meibomian Glands and Other Tear Film Parameters in Junior High School Students. Cornea. 2017 Aug;36(8):922-6.

24 Donald C, Hamilton L, Doughty M. A quantitative assessment of the location and width of Marx's line along the marginal zone of the human eyelid. Optom Vis Sci. 2003 Aug; 80(8):564-72.

25 Shaw AJ, Collins MJ, Davis BA, Carney LG Eyelid pressure and contact with the ocular surface. Invest Ophthalmol Vis Sci. $2010 \mathrm{Apr}$; 51(4):1911-7

26 Knop E, Knop N, Zhivov A, Kraak R, Korb DR, Blackie C, et al. The lid wiper and mucocutaneous junction anatomy of the human eyelid margins: an in vivo confocal and histological study. J Anat. 2011 Apr;218(4):44961.

27 Knop N, Korb DR, Blackie CA, Knop E. The lid wiper contains goblet cells and goblet cell crypts for ocular surface lubrication during the blink. Cornea. 2012 Jun;31(6):668-79.

28 Doughty MJ. Morphological features of cells along Marx's line of the marginal conjunctiva of the human eyelid. Clin Exp Optom. 2013 Jan;96(1):76-84.

29 Tian YJ, Liu Y, Zou HD, Jiang YJ, Liang XQ, Sheng MJ, et al. [Epidemiologic study of dry eye in populations equal or over 20 years old in Jiangning District of Shanghai]. Zhonghua Yan Ke Za Zhi. 2009 Jun;45(6):486-91.

30 Hom MM, Martinson JR, Knapp LL, Paugh JR. Prevalence of Meibomian gland dysfunction. Optom Vis Sci. 1990 Sep;67(9):710-2.

31 Viso E, Rodríguez-Ares MT, Abelenda D, Oubiña B, Gude F. Prevalence of asymptomatic and symptomatic meibomian gland dysfunction in the general population of Spain. Invest Ophthalmol Vis Sci. 2012 May;53(6):2601-6.
32 Siak JJ, Tong L, Wong WL, Cajucom-Uy H, Rosman M, Saw SM, et al. Prevalence and risk factors of meibomian gland dysfunction: the Singapore Malay eye study. Cornea. 2012 Nov;31(11):1223-8.

33 Borchman D, Ramasubramanian A, Foulks GN. Human Meibum Cholesteryl and Wax Ester Variability With Age, Sex, and Meibomian Gland Dysfunction. Invest Ophthalmol Vis Sci. 2019 May;60(6):2286-93.

34 Sullivan DA, Sullivan BD, Ullman MD, Rocha EM, Krenzer KL, Cermak JM, et al. Androgen influence on the meibomian gland. Invest Ophthalmol Vis Sci. 2000 Nov;41(12):373242.

35 Suzuki T, Sullivan BD, Liu M, Schirra F, Richards SM, Yamagami H, et al. Estrogen and progesterone effects on the morphology of the mouse meibomian gland. Adv Exp Med Biol. 2002;506(Pt A):483-8.

36 Sullivan BD, Evans JE, Cermak JM, Krenzer KL, Dana MR, Sullivan DA. Complete androgen insensitivity syndrome: effect on human meibomian gland secretions. Arch Ophthalmol. 2002 Dec;120(12):1689-99.

37 Sullivan DA, Sullivan BD, Evans JE, Schirra F, Yamagami H, Liu M, et al. Androgen deficiency, Meibomian gland dysfunction, and evaporative dry eye. Ann N Y Acad Sci. 2002 Jun;966(1):211-22.

38 Golebiowski B, Badarudin N, Eden J, You J, Hampel U, Stapleton F. Does endogenous serum oestrogen play a role in meibomian gland dysfunction in postmenopausal women with dry eye? Br J Ophthalmol. 2017 Feb;101(2): $218-22$.

39 Schirra F, Suzuki T, Richards SM, Jensen RV, Liu M, Lombardi MJ, et al. Androgen control of gene expression in the mouse meibomian gland. Invest Ophthalmol Vis Sci. 2005 Oct; 46(10):3666-75.

40 Suzuki T, Schirra F, Richards SM, Jensen RV, Sullivan DA. Estrogen and progesterone control of gene expression in the mouse meibomian gland. Invest Ophthalmol Vis Sci. 2008 May;49(5):1797-808.

41 Sullivan DA, Jensen RV, Suzuki T, Richards SM. Do sex steroids exert sex-specific and/or opposite effects on gene expression in lacrimal and meibomian glands? Mol Vis. 2009 Aug; 15:1553-72.

42 Duncan DD, Muñoz B, Bandeen-Roche K, West SK; Salisbury Eye Evaluation Project Team. Assessment of ocular exposure to ultraviolet-B for population studies. Photochem Photobiol. 1997 Nov;66(5):701-9.

43 Duncan DD, Muñoz B, Bandeen-Roche K, West SK; Salisbury Eye Evaluation Project Team. Visible and ultraviolet-B ocular-ambient exposure ratios for a general population. Invest Ophthalmol Vis Sci. 1997 Apr;38(5): 1003-11.

44 Guo B, Lu P, Chen X, Zhang W, Chen R. Prevalence of dry eye disease in Mongolians at high altitude in China: the Henan eye study. Ophthalmic Epidemiol. 2010 Aug;17(4):23441.
Measurement of the Lower Lid Margin Thickness
Ophthalmic Res 2021;64:22-27

DOI: $10.1159 / 000507221$ 